\title{
Analysis and Design of an Oscilloscope Deflection System With a Calculable Transfer Function
}

\author{
D. M. Stonebraker* \\ Institute for Basic Standards, National Bureau of Standards, Boulder, Colo. 80302
}

(December 12, 1967)

\begin{abstract}
An oscilloscope deflector is described, which has a calculable transfer function. The deflector is analyzed, leading to its transfer function, in terms of the complex frequency variables. A practical strip line deflector, usable as a pulse standard, is designed; and its frequency response, sensitivity, bandwidth, rise time, step function, and impulse response are calculated. The predicted deflection is down to 70 percent of its $\mathrm{d}$-c value at $2.82 \mathrm{GHz}$, while the step-response 10 to 90 percent rise time is 148 picoseconds. The effects of a drift space on the oscilloscopic display is also discussed. Results are compared with a well-known expression for the parallel plate deflector structure.
\end{abstract}

Key Words: Deflector, drift space, oscilloscope, strip-line, standard, transfer function.

\section{Introduction}

The cathode-ray oscilloscope is the most common and most useful instrument for displaying voltage as a function of time. It directly provides a graphical presentation of a time function, $y(t)$, which is related to the voltage applied to the input terminals of the oscilloscope by the oscilloscope transfer function, $H(\omega)$, providing we assume the oscilloscope is a linear device. This concept is depicted by figure 1 .

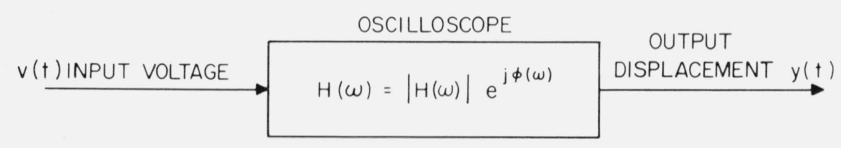

FIGURE 1. Linear system representation of oscilloscope.

When using an oscilloscope to measure a sinusoidal input voltage, we generally assume or require that the oscilloscope be calibrated so that the "true" amplitude of the sinusoid may be read directly from the display. This calibration can be made by measuring the oscilloscope amplitude response, $|H(\omega)|$, from d-c to a suitable high frequency and either adjusting the instrument to read correctly or providing appropriate calibration data to correct the display amplitude. If on the other hand, the input voltage is a pulse or a pulse train, with high harmonic content, the oscilloscope must be calibrated to cor-

*Present Address: 3361 Vista Drive, Boulder, Colo. 80302

'If a standard pulse is available, it may also be used to calibrate the oscilloscope for pulse displays. rect for both amplitude and phase distortion. ${ }^{1}$ This requires measuring both $|H(\omega)|$ and $\varphi(\omega)$, i.e., the amplitude and phase response of the oscilloscope, from d-c to many gigahertz. This is a formidable task owing to the lack of voltage standards above 4. $\mathrm{GHz}$ and the lack of a technique for measuring phase distortion. A suitable alternative is to calculate $H(\omega)$ from knowledge of the physical characteristics of the oscilloscope and verify the calculations, insofar as possible, by sinusoidal measurements. Once $H(\omega)$ is found, an input pulse may be calculated from a knowledge of the output deflection, $y(t)$, by either Fourier series or integral methods.

\section{Design Considerations}

A system analysis of a typical oscilloscope, either real time or sampling, is complicated by the nature of the input probe and amplifiers and by the deflector used. Also the requirement of linearity must be satisfied for a linear analysis. With these criteria in mind, it was decided to select for analysis and design an oscilloscope with a direct feed (no amplifiers), feed through (no probe), $50-\Omega$, real time (nonsampling) deflector system. It was further decided to avoid slow wave (traveling wave) structure for reasons to be discussed next.

Owaki $[1]^{2}$ designed and analyzed a traveling wave cathode-ray tube and obtained a mathematical expression relating spot displacement to the input voltage and geometry of this tube. Hollmann [2] analyzed an elementary parallel plate deflector sys- 
tem and obtained the familiar deflector sensitivity relationship for sinusoidal inputs of the form ${ }^{3}$

$$
\text { relative dynamic sensitivity }=\frac{\sin \omega \tau / 2}{\omega \tau / 2}
$$

where $\omega$ is the angular frequency of the input voltage and $\tau$ is the time taken for the electron to pass between the plates. Talbot [3] considered the same problem and applied convolution integral methods in order to find various pulse response characteristics of the elementary deflector. He applied the results to a direct feed, feed through, slow wave helical deflector. The results of these three workers analyses could be used to write approximate scope transfer functions for their oscilloscopes. However, in the case of Owaki's and Talbot's deflectors, some uncertainties in the transfer function would be difficult to estimate. These uncertainties stem from the effects of coupling and field pertubations between loops of the slow wave structures and resulting specification of transit time. Because we want to avoid significant uncertainties of this type, slow wave structures have been eliminated from consideration. The elementary parallel plate structure could be used, but its sensitivity is extremely small and it is difficult to terminate in its characteristic impedance. Lee [4] showed that this structure can, however, be used in a microoscillograph with sufficient sensitivity for pulse work. Nahman [5] gives an excellent survey of pulse oscilloscopes with a very complete list of references.

Keeping all the criteria mentioned above in mind, as well as Lee's microtechniques, the vacuumfilled strip-line deflector shown in figure 2 was selected.

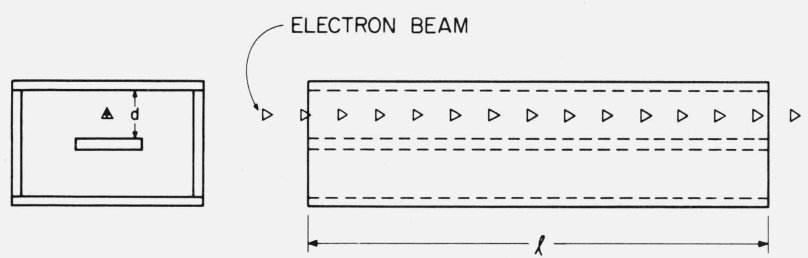

Figure 2. Strip-line deflector.

The electron beam travels between the two conductors in the region where fields, as shown by Brooke [6], are almost uniform if the center conductor is made sufficiently wide.

In order to make a reasonably simple analysis of the relationship between a voltage applied between the two conductors and the resultant deflection of the electron beam, the following design criteria and/or assumptions were made:

a. The deflector characteristic impedance is $50 \Omega$

\footnotetext{
${ }^{2}$ Figures in brackets indicate the literature references on page

${ }^{3}$ Hollmann's result does not include the small displacement within the deflector space. It considers only the transverse velocity change within the deflector and the effects of the drift space. Also Hollmann assumes the electromagnetic field between the plates is spacially invariant and ignores the traveling wave nature of the voltage applied to the plates cially invariant and ignores the traveling wave nature of the vo
and the attendant reflections at the open ends of the plates.
}

and can be terminated in $50 \Omega$ so that any standing waves are negligible.

b. The electric fields in the region where the electron beam travels are uniform, i.e., not a function of vertical position.

c. The fringe fields at the ends of the deflector are negligible.

d. Energy transfer from the beam to the field is negligible, i.e., the beam density is small.

e. Only the TEM mode is propagated down the deflector.

f. The conductor and dielectric losses are negligible.

g. The length of the deflector is sufficiently short and the magnetic forces sufficiently small so the effect of the magnetic fields on the electron displacement and on the longitudinal velocity may be neglected. Hutter [7] and Spangenberg [8] discuss the magnetic effects involved in this assumption.

$\mathrm{h}$. The electron beam radius is assumed to be small compared with the distance between the deflector conductors and compared with the displacement of the electrons due to the voltage applied to the deflector.

\section{Analysis of the Deflection System}

We desire to obtain a transfer function that relates the input voltage to the resultant deflection of the electron beam at the end of the deflector. Since we wish to find the entire effect of the deflection system, we will consider initially only the displacement in the deflector and take up the effects of any possible drift space later. We note that the electrons travel at a velocity less than the velocity of propagation of the electromagnetic wave so that an individual electron slips behind the electromagnetic wave as the electron and the wave travel down the deflector. As a result, the system might be referred to as a slipping traveling wave structure. The slip will necessarily have to be accounted for in the final expression.

A modified phasor approach will be used to find the oscilloscope transfer function. This involves finding the sinusoidal steady state output of the deflector

$$
y(t)=|H(\omega)| V_{m} \cos [\omega t+\varphi(\omega)]
$$

caused by the sinusoidal input voltage

$$
v(t)=V_{m} \cos \omega t .
$$

Once (2) is found, the system transfer function can be written directly since it is

$$
H(\omega)=|H(\omega)| e^{j \varphi(\omega)} .
$$

The output and the input are related through two physical phenomena, which occur simultaneously. These are the force and the resultant acceleration on the electrons due to the electromagnetic field and the effect of the traveling wave nature of the field. First, considering the force due to the electric field and using 


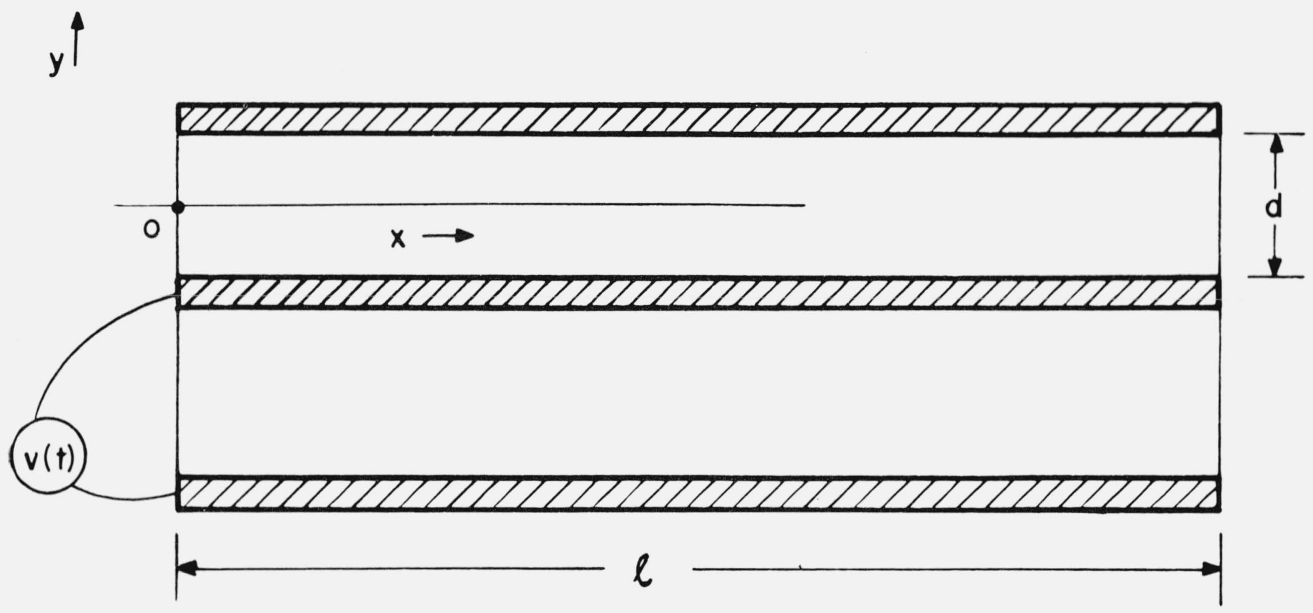

Figure 3. Cross-sectional representation of deflector.

the Lorentz force expression and Newton's Second Law, we get

$$
F_{E}=e E
$$

and

$$
\frac{d^{2} y}{d t^{2}}=\frac{e}{m} E,
$$

where $F_{E}$ is the force in the $y$ direction due to the electric field $E$ in the $y$ direction, and $y$ is the displacement of the electron in the $y$ direction; $e$ and $m$ are the charge and mass of the electron. The coordinate system used is shown in figure 3 .

From the solution of the wave equation, we know that the electric field is a function of the position variable $x$ and time $t$ and is of the functional form

$$
E(x, t)=\frac{v\left(t-\frac{x}{v_{p}}\right)}{d}
$$

for a uniform field, where $d$ is the distance between the conductors, $v$ is the input voltage and $v_{p}$ is the velocity of the electromagnetic wave, which is, throughout the discussion to follow, the velocity of light in a vacuum. The $x$ displacement of the electron is a function of the velocity in the $x$ direction, $v_{x}$. If we assume the electron velocity is constant,

$$
v_{x}=\frac{d x}{d t}=\text { constant }
$$

Then,

$$
x=v_{x}\left(t-t_{0}\right)
$$

where $t_{0}$ is the time an electron enters the deflector at $x=0$. If we substitute (9) into (7) and (7) into (6), we get

$$
\frac{d^{2} y}{d t^{2}}=\frac{e}{m d} v\left[t-\frac{v_{x}}{v_{p}}\left(t-t_{0}\right)\right]
$$

If we specify the input voltage as a sinusoid,

$$
v(t)=V_{m} \cos \omega t,
$$

then (10) becomes

$$
\frac{d^{2} y}{d t^{2}}=K V_{m} \cos \omega\left[t-\frac{v_{x}}{v_{p}}\left(t-t_{0}\right)\right]
$$

where $K=e / m d$. The initial conditions at $x=0$ are

$$
t=t_{0}, y=0, \frac{d y}{d t}=0
$$

By integrating (12) twice and satisfying the initial conditions, we find

$y(t)=\frac{K V_{m}}{\omega^{2} \sigma^{2}}\left[\cos \omega t_{0}-\left(t-t_{0}\right) \omega \sigma \sin \omega t_{0}\right.$

$$
\left.-\cos \omega\left(\sigma t+t_{0} \frac{v_{x}}{v_{p}}\right)\right]
$$

where

$$
\sigma=\frac{v_{p}-v_{x}}{v_{p}}
$$

Sigma is a measure of the slip of the electron with respect to the electromagnetic field. Commonly, electron velocities are described by a ratio $\beta=v_{x} / v_{p}$. The slip parameter $\sigma$ is seen to be $\sigma=1-\beta$. Examination of (14) shows the electrons follow a straight-line path with a sinusoid superposed, oscillating at an angular frequency $\omega^{\prime}=\sigma \omega$, the slip frequency. If we could make $\beta$ approach one by increasing $v_{x}$, the slip frequency would approach zero, and we would have a true traveling wave structure as described by Owaki [1] and Talbot [3]. 
Equation (14) is plotted in figure 4. It shows the normalized displacement

$\frac{y}{K V_{m} / \omega^{2} \sigma^{2}}=\cos \omega t_{0}-\left(t-t_{0}\right) \omega \sigma \sin \omega t_{0}$

$$
-\cos \omega\left(\sigma t+t_{0} \frac{v_{x}}{v_{p}}\right)
$$

plotted versus $\omega\left(t-t_{0}\right)$. Each curve represents the path an electron follows after entering the deflector at the indicated value of $\omega t_{0}$. Since a practical deflector would only be, at most, a few centimeters long, i.e., fractional nanosecond transit time, the oscillatory character of the electrons paths would normally not be observed except for extremely large $\omega$.

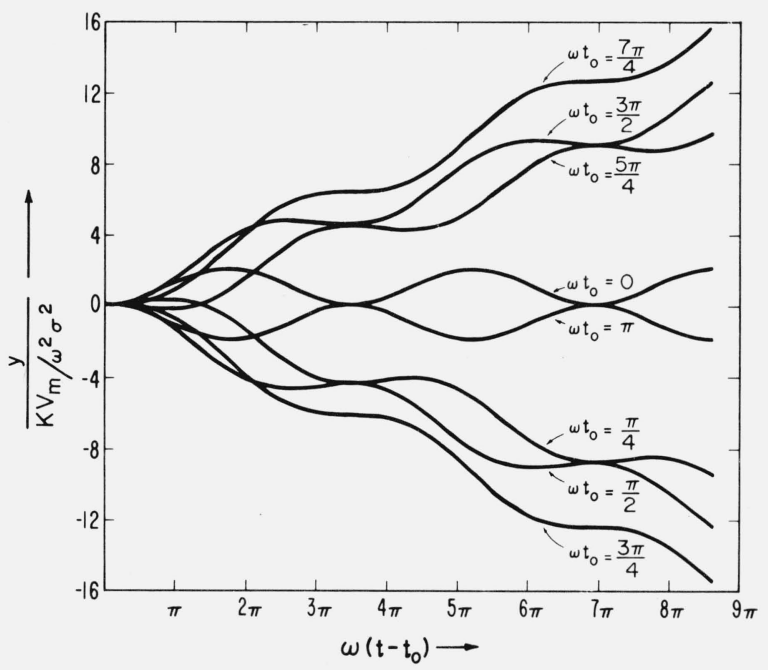

FIGURE 4. Electron path within deflector versus $\mathrm{w}\left(\mathrm{t}-\mathrm{t}_{0}\right)$ for sinusoidal input.

Transit time of the electron, $\tau$, is given by

$$
\tau=\frac{l}{v_{x}}
$$

thus $t=t_{0}+\tau$ at the end of the deflector. If we solve (14) for displacement at the end of the deflector, we get

$y\left(t_{0}\right)=\frac{K V_{m}}{\omega^{2} \sigma^{2}}\left[\cos \omega t_{0}-\tau \omega \sigma \sin \omega t_{0}\right.$

$$
\left.-\cos \left(\omega \sigma \tau+\omega t_{0}\right)\right] .
$$

Equation (16) shows the displacement at the end of the deflector is a sinusoidal function of the time the electrons enter the deflector, $t_{0}$. Since we desire the electron displacement as a function of changing $t_{0}$, let us set

$$
t_{0}=t^{\prime}
$$

where $t^{\prime}$ is a variable time at the end of the deflector.
Replacing $t_{0}$ by $t^{\prime}$ yields the sinusoidal steady state response expressed as follows:

$y\left(t^{\prime}\right)=\frac{K V_{m}}{\omega^{2} \sigma^{2}}\left[\cos \omega t^{\prime}-\omega \tau \sigma \sin \omega t^{\prime}\right.$

$$
\left.-\cos \left(\omega t^{\prime}+\omega \tau \sigma\right)\right] .
$$

If we write (18) in phase and magnitude form, we will have the deflector output in the proper form for finding $H(\omega)$ as specified in (2) and (4), providing we properly account for the transit time delay between input and output. The identity

$$
\cos \left(\omega t^{\prime}+\omega \tau \sigma\right)=\cos \omega t^{\prime} \cos \omega \tau \sigma-\sin \omega t^{\prime} \sin \omega \tau \sigma
$$

allows us to write (18) as follows:

$$
\begin{aligned}
y\left(t^{\prime}\right)=\frac{K V_{m}}{\omega^{2} \sigma^{2}}[(1- & \cos \omega \tau \sigma) \cos \omega t^{\prime} \\
& \left.+(\sin \omega \tau \sigma-\tau \omega \sigma) \sin \omega t^{\prime}\right]
\end{aligned}
$$

which may be written as

$$
y\left(t^{\prime}\right)=\frac{K V_{m}}{\omega^{2} \sigma^{2}} \sqrt{A^{2}+B^{2}} \cos \left(\omega t^{\prime}+\varphi\right),
$$

where

$$
\begin{aligned}
& A=(1-\cos \omega \tau \sigma) \\
& B=(\sin \omega \tau \sigma-\tau \omega \sigma) \\
& \varphi=-\tan ^{-1} \frac{B}{A} .
\end{aligned}
$$

Equation (21) provides the necessary information for writing the oscilloscope transfer function except for the problem of accounting for the delay. Without the delay factor, we get from (21)

$$
H^{\prime}(\omega)=\frac{K}{\omega^{2} \sigma^{2}} \sqrt{A^{2}+B^{2}} e^{j \varphi} .
$$

The delay is provided by introducing the delay factor $e^{-j \omega \tau}$ giving the complete oscilloscope function

$$
H(\omega)=\frac{K}{\omega^{2} \sigma^{2}} \sqrt{A^{2}+B^{2}} e^{j(\varphi-\omega \tau)},
$$

where $A, B$, and $\varphi$ are specified in (21).

\section{S-Domain Transfer Function}

While it is true that (23) is the desired oscilloscope transfer function, it offers little insight into character of the system. The $S$-domain (Laplace) transfer func- 
tion, which can be derived from (23), is more compact and is in a more familiar Laplace transform. If we write (23) in rectangular form, with the delay term left in exponential form, it becomes

$$
\begin{aligned}
H(\omega)= & \frac{K}{\omega^{2} \sigma^{2}} e^{-j \omega \tau}(A-j B) \\
& =\frac{K^{\prime} e^{-j \omega \tau}}{\omega^{2}}[(1-\cos \omega \tau \sigma)-j(\sin \omega \tau \sigma-\tau \omega \sigma)],
\end{aligned}
$$

where $K^{\prime}=\frac{K}{\sigma^{2}}$. Writing the trigonometric terms of (24) in exponential form, we get:

$$
\begin{aligned}
H(\omega)=\frac{K^{\prime} e^{-j \omega \tau}}{\omega^{2}}[(1 & \left.-\frac{e^{j \omega \tau \sigma}+e^{-j \omega \tau \sigma}}{2}\right) \\
& \left.-j\left(\frac{e^{j \omega \tau \sigma}-e^{-j \omega \tau \sigma}}{2 j}-\tau \omega \sigma\right)\right] .
\end{aligned}
$$

If we set $\omega=s / j$ and simplify, (25) becomes

$$
H(s)=K^{\prime} e^{-s \tau}\left[\frac{e^{s \tau \sigma}-\tau \sigma s-1}{s^{2}}\right]
$$

or

$$
H(s)=K^{\prime}\left[\frac{e^{-s \tau(1-\sigma)}-\tau \sigma s e^{-\delta \tau}-e^{-s \tau}}{s^{2}}\right] .
$$

Equation (26) provides immediate information on the response of the deflector to various inputs. For example, if the input is a step function, the output is the sum of two parabolic terms and one linear term. One of the parabolic terms and the linear term are both delayed by $\tau$ seconds after the start of the step input; the other parabolic term is delayed $(1-\sigma) \tau$ seconds after the step input. This demonstrates a result which physical reasoning indicates, i.e., that the output response starts at

$$
t=(1-\sigma) \tau=\left(1-\frac{v_{p}-v_{x}}{v_{p}}\right) \tau=\frac{v_{x}}{v_{p}} \tau=\frac{v_{x}}{v_{p}} \cdot \frac{l}{v_{x}}=\frac{l}{v_{p}},
$$

which is the transit time of the electromagnetic wave and not $t=\tau$, the transit time of an electron.

If we set $l / v_{p}=\tau^{\prime}$, an alternate form of the $s$-domain transfer function may be written, giving

$$
H(s)=K^{\prime}\left[\frac{e^{-s \tau^{\prime}}-\tau \sigma s e^{-s \tau}-e^{-s \tau}}{s^{2}}\right] .
$$

\section{The Impulse and Step Function Response}

The impulse response of the deflector can be found by either finding the inverse Fourier transform of (23) or by finding the inverse Laplace transform of (27).
The latter method leads directly to

$$
h(t)=K^{\prime}\left[\left(t-\tau^{\prime}\right) u\left(t-\tau^{\prime}\right)-\tau \sigma u(t-\tau)-(t-\tau) u(t-\tau)\right]
$$

where $u(t-\tau)$ is the unit step function delayed $\tau$ seconds.

In the previous section, we discussed qualitatively the system step function response. Now, we would like to write an expression for the response to the step input voltage, $v(t)=V u(t)$, by taking the inverse Laplace transform of

$$
Y(s)=V(s) H(s)
$$

where

$$
V(s)=\frac{V}{s}
$$

and $H(s)$ is as specified in (26), which yields

$$
\begin{array}{r}
y(t)=K^{\prime} V\left[\frac{\left(t-\tau^{\prime}\right)^{2}}{2} u\left(t-\tau^{\prime}\right)-\tau \sigma(t-\tau) u(t-\tau)\right. \\
\left.-\frac{(t-\tau)^{2}}{2} u(t-\tau)\right]
\end{array}
$$

as the step function response. Figure 5 shows a sketch of the step function response.

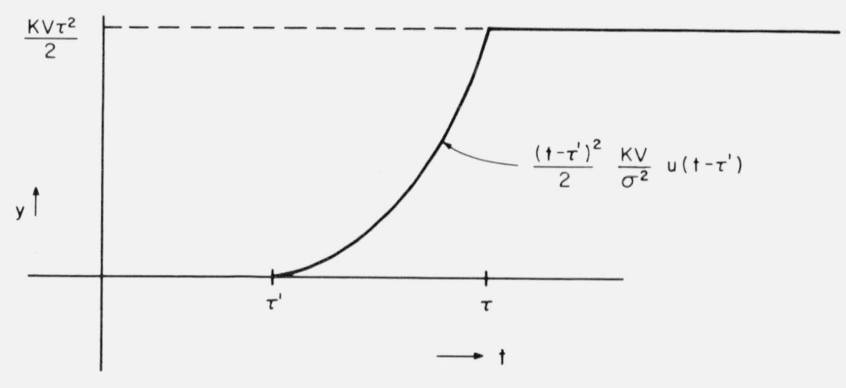

FIGURE 5. Step function response of deflector.

Equation (31) shows that the display at the end of the deflector (due to a step function of voltage applied at the deflector input) starts at $t=\tau^{\prime}$, i.e., the transit time of the electromagnetic wave, and increases parabolically until $t=\tau$, the electron transit time. After $t=\tau$, the displacement becomes the sum of a positive going parabola, a negative going parabola and a negative going ramp. This sum term is simply $K V \tau^{2} / 2$, which is the steady state response and is, as would be expected, the deflection due to a d-c input voltage, $V$. The slip parameter $\sigma=1-v_{x} / v_{p}$ appears in the transit part of the response and cancels out in the steady state part. This is as would be expected since the concept of slip applies to a traveling wave phenomenon and not to a steady d-c voltage applied to the deflector. 


\section{Frequency Response Function}

The frequency response function for the deflector is simply $H(\omega)$ as expressed in (23) where the amplitude response is

$$
|H(\omega)|=\frac{K}{\sigma^{2} \omega^{2}} \sqrt{(1-\cos \omega \tau \sigma)^{2}+(\sin \omega \tau \sigma-\omega \tau \sigma)^{2}}
$$

and the phase response is

$$
\varphi(\omega)=-\omega \tau+\tan ^{-1} \frac{\omega \tau \sigma-\sin \omega \tau \sigma}{1-\dot{\cos } \omega \tau \sigma} .
$$

The linear phase term $\omega \tau$ simply delays all components of the signal an amount $\tau$ and causes no phase distortion; therefore, it is omitted in the plot shown in figure 6 , where the

$$
\text { normalized amplitude }=|H(\omega)| /|H(0)|
$$

and the phase $\varphi(\omega)$ are plotted versus

$$
\text { normalized angular frequency }=\omega \tau \sigma .
$$

The bandwidth of the deflector may be defined as the frequency where the normalized amplitude drops to 0.707 , which occurs at $\omega \tau \sigma \cong 1.12 \pi$, which gives

$$
f_{.707}=\frac{\omega .707}{2 \pi}=\frac{1.12 \pi}{2 \pi \tau \sigma}=\frac{0.56}{\tau \sigma} .
$$

This result shows that the bandwidth is inversely proportional to $\sigma \tau$ where

$$
\sigma \tau=\frac{l}{v_{x}}\left(1-\frac{v_{x}}{v_{p}}\right)=l\left(\frac{1}{v_{x}}-\frac{1}{v_{p}}\right),
$$

which indicates that the bandwidth is proportional to the electron velocity, $v_{x}$, and inversely proportional to the length of the deflector, $l$. In the next section, we see that exactly the inverse is true for deflector sensitivity.

Actually, the concept of bandwidth is of little concern in this discussion since with closed expressions for $|H(\omega)|$ and $\varphi(\omega)$, we may determine exactly the contribution of harmonic components to as high a frequency as we please. Notwithstanding, since bandwidth and its counterpart, rise time, are the usual, but approximate, figures of merit used to describe oscilloscopes, we will give them suitable emphasis.

\section{Design of a Practical Deflector}

In designing a practical deflector of the type shown in figure 3 with a calculable transfer function, we must consider the length, $l$, conductor spacing, $d$, and the velocity of the electrons, $v_{x}$. Since a d-c voltage gives the maximum deflection, as shown in figure 6 , it will be used as the basis for design. The d-c deflection is the steady state value of (31), which is

$$
y(\infty)=\frac{K V}{2} \tau^{2},
$$

where $V$ is the amplitude of the d-c input voltage or

$$
y(\infty)=\frac{K V}{2} \frac{l^{2}}{v_{x}^{2}}=\frac{e V}{2 m d} \frac{l^{2}}{v_{x}^{2}}
$$

where $m$ is the relativistic electron mass, $m_{0} / \sqrt{1-\beta^{2}}$. The sensitivity then, is

Deflector Sensitivity $=\frac{y(\infty)}{V}=\frac{e l^{2} \sqrt{1-\beta^{2}}}{2 m_{0} d v_{x}^{2}} \frac{\text { meter }}{\text { volt }}$.

The symbol $m_{0}$ is the rest mass of the electron, and $\beta$ is the ratio of the velocity of the electron to the velocity of light, which is the same as the $\beta$ discussed in the paragraph following (15) for an air-filled stripline. We must consider the effect of relativity since we want to minimize the slip, i.e., use relatively large electron velocities, in order to increase the bandwidth. This, of course, decreases the sensitivity since (39) shows that the sensitivity is directly proportional to the length squared and inversely proportional to the electron velocity squared if the effect of $\beta^{2}$ is ignored. It also shows that a compromise is necessary between length and conductor spacing in order to optimize the sensitivity and avoid the problem of electrons striking the wall of the deflector before reaching the end. Some practical upper limit of $v_{x}$ and some usable
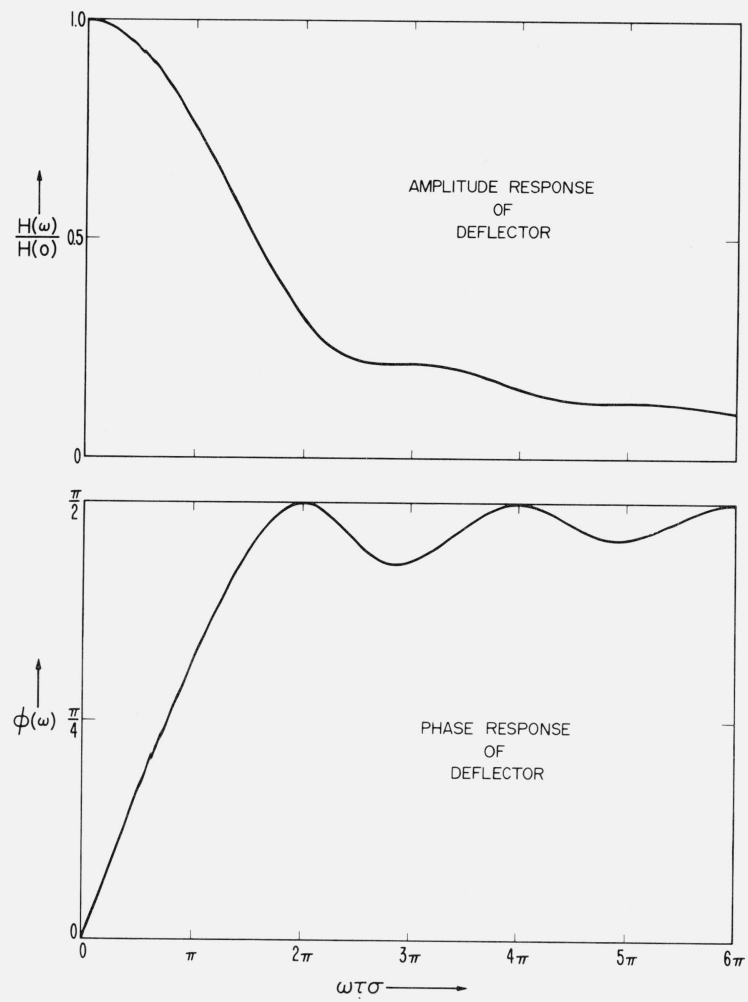

FigURE 6. Amplitude and phase response of deflector. 

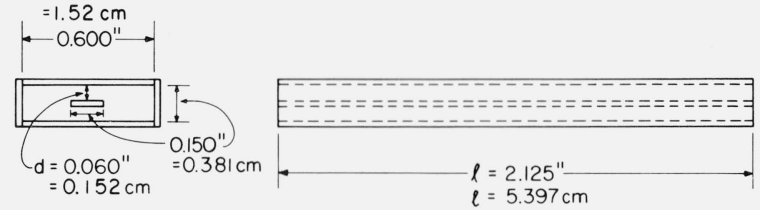

FIGURE 7. Slipping traveling wave deflector $(\tau=429$ ps for $\beta=0.42)$.

lower limit to the deflector sensitivity are the governing criteria in the design, although the other oscilloscope criteria such as light-output intensity, writing speed, and size of screen display are also of primary importance.

An example of a usable deflector, where Lee's [4] microoscillographic techniques must be used, ${ }^{4}$ is shown in figure 7 which is designed for an accelerating potential of $50 \mathrm{kV}$, i.e., $\beta=0.42$. The structure is designed with a characteristic impedance of $50 \Omega$ based on Metcalf's [9] design curves of characteristic impedance for the rectangular strip lines. The calculated deflector sensitivity from (39) is $9.64 \times 10^{-6} \mathrm{~m} / \mathrm{V}$ or about $0.01 \mathrm{~mm} / \mathrm{V}$. The lowest TM mode cutoff wavelength is approximately twice the ground plane spacing according to Howe [10]

$$
\lambda_{\text {СОтм }}=2(0.15)=(0.30) \text { in }=0.662 \mathrm{~cm},
$$

which is equal to a cutoff frequency of $39.3 \mathrm{GHz}$. The lowest TE mode cutoff wavelength equals approximately the mean circumferential path

$$
\begin{aligned}
\lambda_{\text {COTE }}=2(0.15)+\frac{1}{2} \pi(0.15)=\left(2+\frac{1}{2} \pi\right)(0.15) \\
=0.53 \mathrm{in}=1.346 \mathrm{~cm}
\end{aligned}
$$

which is equal to a cutoff frequency of $22.3 \mathrm{GHz}$.

The bandwidth of this design from (36) is

$$
f_{.707}=\frac{0.56}{\tau \sigma}=\frac{0.56}{429 \times 10^{-12} \times 0.48}=2.82 \mathrm{GHz} .
$$

It is interesting in this case, to calculate, using the derived transfer function, the actual rise time of the deflector to a step input. This is easily done using (31). Equation (31) leads to the oscilloscope display shown in figure 5, which has a 10 to 90 percent rise time

$$
t_{r}=\sigma \tau \sqrt{0.9}-\sigma \tau \sqrt{0.1}=0.634 \sigma \tau=148 \mathrm{ps} .
$$

\section{Drift Space Considerations}

Thus far in our discussion, we have considered an oscilloscope with no drift space beyond the deflector. In general, some drift space will be involved; if we include a drift space, the oscilloscope transfer function, (27), must be modified accordingly. As shown in the appendix, the total oscilloscope transfer function in the $S$-domain is

4 Lee [4] describes the nature of the appropriate beam size and focusing techniques as well as suitable methods of recording for a microoscillographic deflector.

$$
H_{T}(S)=H(S) e^{-\tau^{\prime \prime}} s+\frac{\tau^{\prime \prime} K}{\sigma} \frac{e^{-s\left(\tau^{\prime}+\tau^{\prime \prime}\right)}-e^{-\left(\tau+\tau^{\prime \prime}\right)} s}{S}
$$

where $\tau^{\prime \prime}$ is the transit time of the electrons in the drift space

$$
\tau^{\prime \prime}=\frac{L}{v_{x}}
$$

where $L$ is the length of the drift space. The drift space has the effect of increasing the sensitivity. D-C sensitivity, for example, is increased by $2 L / l \times 100$ percent. Thus, if $L=l$, the deflector sensitivity is increased by 200 percent.

In the appendix the expression for the relative sensitivity of the slipping strip line deflector is developed for the drift space contribution of displacement only, i.e., the deflection within the deflector space is ignored. The result is shown in $(\mathrm{A}-12)$.

$$
\frac{|Y(\omega)|}{|Y(0)|}=\frac{\sin \frac{\sigma \omega \tau}{2}}{\frac{\sigma \omega \tau}{2}} .
$$

It is interesting to compare this result with Hollmann's expression for parallel plate deflector given in (1).

$$
\frac{|Y(\omega)|}{|Y(0)|}=\frac{\sin \frac{\omega \tau}{2}}{\frac{\omega \tau}{2}} .
$$

We see that the results are identical except for the slip factor $\sigma$.

Hollmann's result is based on the assumption of a space invariant electromagnetic field within the deflector while (A-12) includes the effect of a slipping traveling wave. We see, since

$$
\sigma=\frac{v_{p}-v_{x}}{v_{p}},
$$

that under Hollmann's assumption, i.e., infinite wave velocity, the slip approaches one and (A-12) approaches (1) as it should if this work and Hollmann's results are to be compatible.

\section{Conclusion}

Knowledge of the oscilloscope transfer function makes it possible to predict the response characteristics of the oscilloscope to the extent that the physical parameters in the expression can be measured and to the extent that the assumptions employed lead to errors in the calculated transfer function. Uncertainties of these types can be held to a minimum by careful construction and measurement methods. Also, the accuracy of the transfer function can be approxi- 
mately verified experimentally from sinusoidal measurements. A thorough error analysis would contribute to knowledge of the magnitude of uncertainties in the oscilloscope transfer function.

Once the transfer function and its uncertainties are known, it can be used to calculate the actual time function of either a periodic or nonperiodic input pulse from a knowledge of the output display. An analogto-digital conversion of the output can be used for fast and accurate machine calculations. An instrument of this type, which requires microoscillographic techniques and machine calculations, is not practical for normal laboratory measurements of pulses. It can be used as a standard oscilloscope for measuring pulse parameters such as rise time, overshoot, duration and so forth. It also may be used to determine the input voltage amplitude as a function of time to a stated accuracy.

A significant result is found by comparing the relative sensitivity of the slipping traveling-wave strip-line deflector (for the drift space only) with Hollmann's [2] results for the parallel-plate structure. It is concluded that the strip-line results provide a more general expression for the parallel-plate deflector than Hollmann's expression.

The author acknowledges the many helpful suggestions, counsel, and encouragement received from A. R. Ondrejka, and the careful manuscript preparation by Mrs. Toni Hooper.

\section{References}

[1] K. Owaki, S. Terahata, T. Hada, and T. Nakamura, The traveling-wave cathode-ray tube, Proc. IRE 38, 1172-1180 (Oct. 1950).

[2] H. E. Hollmann, Die Braunsche Rohre bei sehr hohen Frequenzen, Hochfrequenz und Electroakustik 40, 97-103 (Sept. 1932).

[3] R. V. Talbot, and L. M. Johnson, The TW-10 a high writing speed cathode-ray tube with distributed deflection, NRL Report 4377, May 25, 1954.

[4] Gordon M. Lee, A three-beam oscillograph for recording at frequencies up to 10,000 Megacycles, Proc. IRE 34, 121-127 (Mar. 1946).

[5] N. S. Nahman, A Survey of millimicrosecond pulse instrumentation, Bulletin of Engineering \& Architecture No. 43, University of Kansas, 1959, and N. S. Nahman, The measurement of baseband pulse rise times of less than $10^{-9}$ second, Proc. IEEE, 55, 855-864 (June 1967).

[6] R. L. Brooke, C. A. Hoer, and C. H. Love, Inductance and characteristics impedance of strip-transmission line, J. Res. NBS 71C (Engr. and Instr.) No. 1, 59-67 (Jan.-Mar. 1967).

[7] R. G. E. Hutter, Beam and Wave Electronics in Microwave Tubes, ch. 8 (Van Nostrand Company, Inc., Princeton, N.J., 1960).

[8] K. R. Spangenberg, Vacuum Tubes, ch. 6 (McGraw-Hill, 1948).

[9] W. S. Metcalf, Characteristic impedance of rectangular trans mission lines, Proc. IEE 112 , 2033-2039, No. 11 (Nov. 1965).

[10] Harlan G. Howe, Jr., Dielectrically loaded stripline at $18 \mathrm{GHz}$, Microwave Journal, pp. 52-54 (Jan. 1966).

\section{Appendix}

The addition of a drift space whose length is $L$ meters introduces an additional electron displacement, $Y$, such that

$$
Y=y^{\prime} \tau^{\prime \prime}
$$

where $\tau^{\prime \prime}$ is the time the electron is in the drift space, i.e., drift space transit time, and $y^{\prime}$ is the vertical velocity of the electron at the end of the deflector. Assuming the $x$ velocity is constant,

$$
\tau^{\prime \prime}=\frac{L}{v_{x}} .
$$

The total deflection, $y_{T}$, is

$$
y_{T}=\boldsymbol{Y}+y .
$$

Once the electron leaves the deflector, the displacement force due to the traveling electromagnetic field stops, i.e., $y$ becomes constant. Thus to find $y_{T}$, we need only to find the expression for $Y$. This requires that we obtain an expression for the vertical velocity at the end of the deflector. The vertical velocity within the deflector is found by integrating (12) and evaluating the arbitrary constants using (13). The vertical velocity at the end of the deflector, $y^{\prime}$, is found in the same manner as (18) was found, giving

$$
y^{\prime}=\frac{K V_{m}}{\omega \sigma}\left[\sin \left(\omega t^{\prime}+\omega \sigma \tau\right)-\sin \omega t^{\prime}\right]
$$

where $t^{\prime}=t-\left(t_{0}+\tau\right)$ for $t \geqslant t_{0}+\tau$. Equation (A-4) can be written in phase and magnitude form giving

$$
y^{\prime}=\frac{K V_{m}}{\omega \sigma} \omega\left[\sqrt{C^{2}+D^{2}} \cos \left(\omega t^{\prime}-\theta\right)\right]
$$

where

$$
\begin{aligned}
& C=(-1+\cos \omega \sigma \tau) \\
& D=\sin \omega \sigma \tau \\
& \Theta=\operatorname{Tan}^{-1} \frac{-1+\cos \omega \sigma \tau}{\sin \omega \sigma \tau} .
\end{aligned}
$$

The transfer function due to the drift space is then

$$
H_{D}(\omega)=\frac{K \tau^{\prime \prime}}{\omega \sigma} \sqrt{C^{2}+D^{2}} e^{-j \theta}
$$

which must be combined with (23) to give the total transfer function:

$$
H_{T}(\omega)=H(\omega) e^{-j \omega \tau^{\prime \prime}}+\frac{K \tau^{\prime \prime}}{\omega \sigma} \sqrt{C^{2}+D^{2}} e^{-j\left(\theta+\omega \tau^{\prime \prime}+\omega \tau\right)}
$$

where $e^{-j \omega \tau^{\prime \prime}}$ and $e^{-j \omega \tau}$ provides the delay caused by the drift space, and deflector respectively.

The $S$-domain transfer function may be found by setting $\omega=S / j$ in $(\mathrm{A}-7)$ which can be shown to yield

$$
H_{T}(S)=H(S) e^{-\tau^{\prime \prime} s}+\frac{\pi^{\prime \prime} K}{\sigma}\left[\frac{e^{-s\left(\tau^{\prime}+\tau^{\prime \prime}\right)}-e^{-\left(\tau+\tau^{\prime \prime}\right) s}}{S}\right] .
$$


While (A-8) is the transfer function for which we are searching, it is interesting to compare this result for the strip line deflector with the work of Hollmann for the parallel plate deflector, as expressed in eq (1). The comparison can be made by considering only the $Y$ deflection since Hollmann's equation includes only this part of the total deflection. The comparison is made by finding the relative sensitivity, as defined by Hollmann (which is $|Y(\omega)| /|Y(0)|$ ), for the strip line.

The expression for $|Y(\omega)|$ may be found from (A-6) since

or

$$
H_{D}(\omega)=\frac{Y(\omega)}{V(\omega)}
$$

$$
Y(\omega)=H_{D}(\omega) V(\omega) .
$$

Substituting the magnitude of $(A-6)$ into $(A-9)$ gives

$$
|Y(\omega)|=\frac{V_{m} K \tau^{\prime \prime}}{\omega \sigma} \sqrt{(-1+\cos \omega \sigma \tau)^{2}+(\sin \omega \sigma \tau)^{2}}
$$

since $V(\omega)=V_{m} e^{j 0}$, i.e., the input voltage is $V_{m} \cos \omega t$. It can be shown that

$$
Y(0) \simeq K V_{m} \tau \tau^{\prime \prime}
$$

which is the displacement due to a d-c input, $V_{m}$. The desired ratio is then found from $(\mathrm{A}-10)$ and $(\mathrm{A}-11)$, giving

$$
\frac{|Y(\omega)|}{|Y(0)|}=\frac{\sqrt{(-1+\cos \omega \sigma \tau)^{2}(\sin \omega \sigma \tau)^{2}}}{\omega \sigma \tau}
$$

or

$$
\frac{|Y(\omega)|}{|Y(0)|}=\frac{2 \sqrt{\frac{1-\cos \omega \sigma \tau}{2}}}{\omega \sigma \tau}
$$

or finally

$$
\frac{|Y(\omega)|}{|Y(0)|}=\left|\frac{\sin \frac{\omega \sigma \tau}{2}}{\frac{\omega \sigma \tau}{2}}\right| .
$$

(Paper 72C2-271) 